九州大学学術情報リポジトリ

Kyushu University Institutional Repository

\title{
NONPARAMETRIC SEQUENTIAL ESTIMATION OF A MULTIPLE REGRESSION FUNCTION
}

Ahmad, Ibrahim A.

Department of Statistics, Florida State University

Lin, Pi-Erh

Department of Statistics, Florida State University

https://doi.org/10.5109/13104

出版情報: 統計数理研究. 17 (1/2)，pp.63-75，1976-03. Research Association of Statistical Sciences

バージョン：

権利関係 : 


\title{
NONPARAMETRIC SEQUENTIAL ESTIMATION OF A MULTIPLE REGRESSION FUNCTION*
}

\author{
By
}

\author{
Ibrahim A. AHMAD** and Pi-Erh LIN**
}

(Received July 1, 1975)

\section{Introduction.}

Let $\left(\boldsymbol{X}^{\prime}, Y\right)^{\prime}$ be a $(p+1)$-dimensional random vector having the distribution function $(d f) F^{*}$ and the probability density function $(p d f) f^{*}$ with respect to the Lebesgue measure. Let $F$ and $f$ be the $p$-dimensional marginal $d f$ and $p d f$ of $\boldsymbol{X}$ respectively. Let $k(\boldsymbol{x})$ be a known $p d f$ defined on the $p$-dimensional Euclidean space $R^{p}$ such that

$$
\begin{gathered}
\sup _{\boldsymbol{u} \in R^{p}} k(\boldsymbol{u})<\infty, \quad \text { and } \\
\|\boldsymbol{u}\| k(\boldsymbol{u}) \rightarrow 0 \quad \text { as } \quad\|\boldsymbol{u}\|=\left(\sum_{i=1}^{p} u_{i}^{2}\right)^{1 / 2} \rightarrow \infty .
\end{gathered}
$$

Let $\left\{a_{n}\right\}$ be a sequence of monotone decreasing positive real numbers converging to zero as $n$ tends to infinity. Further conditions on the convergence rate for the sequence $\left\{a_{n}\right\}$ will be needed throughout the study. They are listed as follows:

$$
\begin{gathered}
n a_{n}^{p} \rightarrow \infty, \quad \text { as } n \rightarrow \infty ; \\
n a_{n}^{p+4} \rightarrow 0, \quad \text { as } n \rightarrow \infty ; \\
\frac{1}{n} \sum_{j=1}^{n}\left(\frac{a_{n}}{a_{j}}\right)^{p} \rightarrow \alpha(0 \leqq \alpha \leqq 1), \quad \text { as } n \rightarrow \infty ; \text { and } \\
\sum_{n=1}^{\infty} \exp \left(-\varepsilon n a_{n}^{p}\right)<\infty \quad \text { for any } \varepsilon>0 .
\end{gathered}
$$

Based on a random sample $\left(\boldsymbol{X}_{1}^{\prime}, Y_{1}\right)^{\prime}, \cdots,\left(\boldsymbol{X}_{n}^{\prime}, Y_{n}\right)^{\prime}$ from $F^{*}$ we wish to estimate the multiple regression function

$$
m(\boldsymbol{x})=E(Y \mid \boldsymbol{X}=\boldsymbol{x})=h(\boldsymbol{x}) / f(\boldsymbol{x}),
$$

where

$$
h(\boldsymbol{x})=\int_{R} y f^{*}(\boldsymbol{x}, y) d y .
$$

It will be assumed throughout the study that $m(\boldsymbol{x})<\infty$. A nonparametric estimate

* Research supported in part by The National Institute of General Medical Sciences through Training Grant 5T01 GM-913.

** Department of Statistics, Florida State University, Tallahassee, Florida, U.S.A. 
of $m(\boldsymbol{x})$ may be given by

$$
m_{n}(\boldsymbol{x})=h_{n}(\boldsymbol{x}) / f_{n}(\boldsymbol{x})
$$

where

and

$$
f_{n}(\boldsymbol{x})=\frac{1}{n a_{n}^{p}} \sum_{j=1}^{n} k\left(\frac{\boldsymbol{x}-\boldsymbol{X}_{j}}{a_{n}}\right)
$$

with

$$
h_{n}(\boldsymbol{x})=\frac{1}{n a_{n}^{p}} \sum_{j=1}^{n} Y_{j} k\left(\frac{\boldsymbol{x}-\boldsymbol{X}_{j}}{a_{n}}\right),
$$

$$
k\left(\frac{\boldsymbol{u}}{a_{n}}\right)=k\left(\frac{u_{1}}{a_{n}}, \cdots, \frac{u_{p}}{a_{n}}\right) .
$$

The density estimate $f_{n}(\boldsymbol{x})$ was considered by Cacoullos (1966).

For the simple regression case, i. e., $p=1$, the estimate $m_{n}(\boldsymbol{x})$ was proposed by Nadaraya (1964) and independently by Watson (1964). Schuster (1972) showed that, for this case, the asymptotic distribution of $\left(n a_{n}\right)^{1 / 2}\left[m_{n}\left(x_{1}\right)-m\left(x_{1}\right), \cdots, m_{n}\left(x_{q}\right)-m\left(x_{q}\right)\right]$ is $q$-variate normal with mean vector $0 \in R^{q}$ and diagonal covariance matrix $C$ where the $i$-th element is $C_{i i}=\operatorname{Var}\left(Y \mid X=x_{i}\right) \int_{R} k^{2}(u) d u / f\left(x_{i}\right), i=1, \cdots, q$.

Yamato (1971) proposed a sequential estimate for $f(\boldsymbol{x})$ as follows :

$$
\hat{f}(\boldsymbol{x})=\frac{1}{n} \sum_{j=1}^{n} \frac{1}{a_{j}^{p}} k\left(\frac{\boldsymbol{x}-\boldsymbol{X}_{j}}{a_{j}}\right) .
$$

The estimate $\hat{f}(\boldsymbol{x})$ has an advantage over $f_{n}(\boldsymbol{x})$ in that when the sample size $n$ increases only the additional terms need to be computed and hence $f(\boldsymbol{x})$ may be estimated sequentially. With the same spirit, we propose a nonparametric sequential estimate for the multiple regression $m(\boldsymbol{x})$ as follows:

$$
\hat{m}(\boldsymbol{x})=\hat{h}(\boldsymbol{x}) / \hat{f}(\boldsymbol{x}),
$$

where $\hat{f}(\boldsymbol{x})$ is given by (1.11) and

$$
\hat{h}(\boldsymbol{x})=\frac{1}{n} \sum_{j=1}^{n} \frac{1}{a_{j}^{p}} Y_{j} k\left(\frac{\boldsymbol{x}-\boldsymbol{X}_{j}}{a_{j}}\right) .
$$

The purpose of this paper is to investigate important large sample properties of $\hat{m}(\boldsymbol{x})$. In Section 2, weak and strong (pointwise as well as uniform) consistencies of $\hat{m}(\boldsymbol{x})$ are shown. Weak consistency is established under conditions of Yamato's (1971) Theorem 2, while strong consistency may be proved using the technique first introduced by van Ryzin (1969) and later used by Davies (1973) in proving the consistency of $\hat{f}(\boldsymbol{x})$. Finally the joint asymptotic normality of $\left(n a_{n}^{p}\right)^{1 / 2}\left[\hat{m}\left(\boldsymbol{x}_{1}\right)-m\left(\boldsymbol{x}_{1}\right), \cdots, \hat{m}\left(\boldsymbol{x}_{q}\right)-m\left(\boldsymbol{x}_{q}\right)\right]$ is shown in Section 3.

Throughout the study the following well known result will be used repeatedly. This was also quoted by Yamato (1971, Lemma 2).

LEMMA A. If a sequence of functions $\left\{\delta_{n}(\boldsymbol{x})\right\}$ converges to a function $\delta(\boldsymbol{x})$ at a point $\boldsymbol{x}$ as $n \rightarrow \infty$, then $n^{-1} \sum_{j=1}^{n} \delta_{j}(\boldsymbol{x}) \rightarrow \delta(\boldsymbol{x})$ as $n \rightarrow \infty$. If a sequence of functions $\left\{\delta_{n}(\boldsymbol{x})\right\}$ is uniformly bounded in $R^{p}$ and converges to a bounded function $\delta(\boldsymbol{x})$, as $n \rightarrow \infty$, uni- 
formly in $R^{p}$, then $n^{-1} \sum_{i=1}^{n} \delta_{j}(\boldsymbol{x}) \rightarrow \delta(\boldsymbol{x})$, as $n \rightarrow \infty$, uniformly in $R^{p}$.

\section{Consistency of the multiple regression estimate.}

In this section under some regularity conditions the pointwise consistency (Theorems 1 and 2) as well as the uniform consistency (Theorems 3 and 4) of $\hat{m}(\boldsymbol{x})$ will be established. Preliminary lemmas necessary to carry out the proof of each theorem shall precede its actual statement.

LEMmA 1. If $k$ satisfies (1.1) and (1.2), then at each continuity point $\boldsymbol{x}$ of $h$

$$
E \hat{h}(\boldsymbol{x}) \rightarrow h(\boldsymbol{x}), \quad \text { as } n \rightarrow \infty \text {. }
$$

Proof. From Lemma A it suffices to show that $a_{n}^{-p} E\left\{Y_{n} k\left[\left(\boldsymbol{x}-\boldsymbol{X}_{n}\right) / a_{n}\right]\right\} \rightarrow h(\boldsymbol{x})$, at each continuity point $\boldsymbol{x}$ of $h$. Now

$$
\begin{aligned}
a_{n}^{-p} E\left[Y_{n} k\left(\frac{\boldsymbol{x}-\boldsymbol{X}_{n}}{a_{n}}\right)\right] & =a_{n}^{-p} \int_{R} \int_{R} y k\left(\frac{\boldsymbol{x}-\boldsymbol{u}}{a_{n}}\right) f^{*}(\boldsymbol{u}, y) d \boldsymbol{u} d y \\
& =a_{n}^{-p} \int_{R^{p}} E\left[Y k\left(\frac{\boldsymbol{x}-\boldsymbol{u}}{a_{n}}\right) \mid \boldsymbol{X}=\boldsymbol{u}\right] f(\boldsymbol{u}) d \boldsymbol{u} \\
& =a_{n}^{-p} \int_{R^{p}} k\left(\frac{\boldsymbol{x}-\boldsymbol{u}}{a_{n}}\right) h(\boldsymbol{u}) d \boldsymbol{u} \\
& =\int_{R^{p}} k(\boldsymbol{v}) h\left(\boldsymbol{x}-a_{n} \boldsymbol{v}\right) d \boldsymbol{v} \rightarrow h(\boldsymbol{x}),
\end{aligned}
$$

as $n \rightarrow \infty$. The third equality is obtained by the substitution of (1.7), the fourth equality by the transformation $\boldsymbol{v}=(\boldsymbol{x}-\boldsymbol{u}) / a_{n}$, and the final result by the facts that $h$ is continuous at $\boldsymbol{x}$ and $k$ is a pdf.

LEMma 2. Assume that conditions of Lemma 1 and (1.3) hold. If

$$
E\left(Y^{2} \mid \boldsymbol{X}=\boldsymbol{x}\right)<\infty,
$$

then, at each continuity point $\boldsymbol{x}$ of $h$,

$$
\operatorname{Var}[\hat{h}(\boldsymbol{x})] \rightarrow 0, \quad \text { as } n \rightarrow \infty .
$$

Proof. Note that

$$
\begin{aligned}
\operatorname{Var}[\hat{h}(\boldsymbol{x})]= & n^{-2} \sum_{j=1}^{n} a_{j}^{-2 p} \operatorname{Var}\left[Y_{j} k\left(\frac{\boldsymbol{x}-\boldsymbol{X}_{j}}{a_{j}}\right)\right] \\
= & n^{-2} \sum_{j=1}^{n} a_{j}^{-2 p} \int_{R} \int_{R^{p}} y^{2} k^{2}\left(\frac{\boldsymbol{x}-\boldsymbol{u}}{a_{j}}\right) f^{*}(\boldsymbol{u}, y) d \boldsymbol{u} d y \\
& \quad-n^{-2} \sum_{j=1}^{n} a_{j}^{-2 p}\left[\int_{R} \int_{R} p k\left(\frac{\boldsymbol{x}-\boldsymbol{u}}{a_{j}}\right) f^{*}(\boldsymbol{u}, y) d \boldsymbol{u} d y\right]^{2} \\
\leqq & n^{-2} \sum_{j=1}^{n} a_{j}^{-2 p} \int_{R} \int_{R^{p}} y^{2} k^{2}\left(\frac{\boldsymbol{x}-\boldsymbol{u}}{a_{j}}\right) f^{*}(\boldsymbol{u}, y) d \boldsymbol{u} d y \\
= & n^{-2} \sum_{j=1}^{n} a_{j}^{-2 p} \int_{R^{p}} k^{2}\left(\frac{\boldsymbol{x}-\boldsymbol{u}}{a_{j}}\right) g(\boldsymbol{u}) d \boldsymbol{u},
\end{aligned}
$$

where 


$$
g(\boldsymbol{u})=E\left(Y^{2} \mid \boldsymbol{X}=\boldsymbol{u}\right) f(\boldsymbol{u}) .
$$

But, as in Lemma 1, $a_{n}^{-p} \int_{R^{p}} k^{2}\left[(\boldsymbol{x}-\boldsymbol{u}) / a_{n}\right] g(\boldsymbol{u}) d \boldsymbol{u} \rightarrow g(\boldsymbol{x}) \int_{R^{p}} k^{2}(\boldsymbol{u}) d \boldsymbol{u}<\infty$, as $n \rightarrow \infty$. Hence

$$
\left(n a_{n}^{2 p}\right)^{-1} \int_{R^{p}} k^{2}\left(\frac{\boldsymbol{x}-\boldsymbol{u}}{a_{j}}\right) g(\boldsymbol{u}) d \boldsymbol{u} \rightarrow 0,
$$

as $n \rightarrow \infty$, and the lemma follows by an application of Lemma $\mathrm{A}$.

The weak pointwise consistency of $\dot{m}(\boldsymbol{x})$ is obtained in the following:

THEorem 1. If $k$ satisfies Conditions (1.1) and (1.2), and if $\left\{a_{n}\right\}$ satisfies Condition (1.3), then, at each continuity point $\boldsymbol{x}$ of $f$ and $h$ for which $f(\boldsymbol{x})>0$,

in probability, as $n \rightarrow \infty$.

$$
\hat{m}(\boldsymbol{x}) \rightarrow m(\boldsymbol{x}),
$$

Proof. It follows from Lemmas 1 and 2 that $\hat{h}(\boldsymbol{x}) \rightarrow h(\boldsymbol{x})$ in probability, as $n \rightarrow \infty$, at each continuity point $\boldsymbol{x}$ of $h$. Also $\hat{f}(\boldsymbol{x}) \rightarrow f(\boldsymbol{x})>0$ in probability, as $n \rightarrow \infty$, at each continuity point $\boldsymbol{x}$ of $f$ (Corollary 4, Yamato (1971)). The theorem follows by an application of Slutsky's lemma.

Condition (1.3), assumed in Theorem 1, is not sufficient for the strong consistency which is to be established in the next theorem. A somewhat stronger condition on the convergence rate of $\left\{a_{n}\right\}$ will be needed.

THEOREm 2. Suppose that $Y$ and $f(\boldsymbol{x})$ are both bounded, that $k$ satisfies Conditions (1.1) and (1.2), and that $\left\{a_{n}\right\}$ satisfies Conditions (1.5) and (1.6). If (2.1) holds, then, at each continuity point $\boldsymbol{x}$ of $f, h$ and $g$ for which $f(\boldsymbol{x})>0$,

$$
\hat{m}(\boldsymbol{x}) \rightarrow m(\boldsymbol{x}),
$$

with probability one (w.p.1), as $n \rightarrow \infty$.

Proof. Similar to Theorem 1 , we will need to show that, as $n \rightarrow \infty$,

$$
\hat{h}(\boldsymbol{x}) \rightarrow h(\boldsymbol{x}) \text {, w. p. } 1,
$$

and

$$
\hat{f}(\boldsymbol{x}) \rightarrow f(\boldsymbol{x}), \text { w. p. } 1 .
$$

From Lemma 1 above and Corollary 2 of Yamato (1971) it suffices to show that $|\hat{h}(\boldsymbol{x})-E \hat{h}(\boldsymbol{x})| \rightarrow 0$ and $|\hat{f}(\boldsymbol{x})-E \hat{f}(\boldsymbol{x})| \rightarrow 0$ w. p. 1, as $n \rightarrow \infty$. We shall only prove the first assertion, the second can be proved similarly. Since $\hat{h}(\boldsymbol{x})=n^{-1} \sum_{j=1}^{n} a_{j}^{-p} Y_{j} k\left[\left(\boldsymbol{x}-\boldsymbol{X}_{j}\right) / a_{j}\right]$, $n \hat{h}(\boldsymbol{x})=\sum_{j=1}^{n} R_{j}(\boldsymbol{x})$ is the sum of $n$ independent random variables where, for $j=1, \cdots, n$,

$$
R_{j}(\boldsymbol{x})=\frac{1}{a_{j}^{p}}\left\{Y_{j} k\left(\frac{\boldsymbol{x}-\boldsymbol{X}_{j}}{a_{j}}\right)-E Y_{j} k\left(\frac{\boldsymbol{x}-\boldsymbol{X}_{j}}{a_{j}}\right)\right\} .
$$

Note that $R_{j}(\boldsymbol{x})$ is bounded, i. e.,

$$
\max _{1 \leqq j \leqq n}\left|R_{j}(\boldsymbol{x})\right| \leqq M<\infty .
$$

Thus it follows from the Bernstein inequality [see, e.g., Bennett (1962)] that, for any $t>0$, 


$$
P\left[\left|\sum_{j=1}^{n} R_{j}(\boldsymbol{x})\right|>t \sigma_{n}\right] \leqq 2 \exp \left\{-\frac{t^{2}}{2\left(1+\frac{M t}{3 \sigma_{n}}\right)}\right\},
$$

where

$$
\boldsymbol{\sigma}_{n}^{2}=\operatorname{Var}\left[\sum_{j=1}^{n} R_{j}(\boldsymbol{x})\right]
$$

Therefore, for any $\varepsilon>0$,

$$
P[|\hat{h}(\boldsymbol{x})-E \hat{h}(\boldsymbol{x})|>\varepsilon] \leqq 2 \exp \left\{-\frac{\varepsilon^{2} n}{2\left(\frac{\sigma_{n}^{2}}{n}+\frac{\varepsilon M}{3}\right)}\right\} .
$$

The result (2.3) will be proved if we can show that the infinite sum of the right hand side of (2.5) is finite. In view of Condition (1.6), this will be accomplished by showing that

$$
\frac{a_{n}^{p} \sigma_{n}^{2}}{n} \rightarrow \alpha g(\boldsymbol{x}) \int_{R^{p}} k^{2}(\boldsymbol{u}) d \boldsymbol{u}<\infty,
$$

as $n \rightarrow \infty$, where $\alpha$ is defined by (1.5) and $g(\boldsymbol{x})$ by (2.2). Note that

$$
\frac{a_{n}^{p} \sigma_{n}^{2}}{n}=\frac{a_{n}^{p}}{n} \sum_{j=1}^{n} \frac{1}{a_{j}^{2 p}} \operatorname{Var}\left[Y_{j} k\left(\frac{\boldsymbol{x}-\boldsymbol{X}_{j}}{a_{j}}\right)\right],
$$

where the $j$-th term in the summand (multiplied by $a_{j}^{p}$ ) is equal to

$$
\begin{gathered}
\frac{1}{a_{j}^{p}} \int_{R^{p}} k^{2}\left(\frac{\boldsymbol{x}-\boldsymbol{u}}{a_{j}}\right) g(\boldsymbol{u}) d \boldsymbol{u}-\frac{1}{a_{j}^{p}}\left[\int_{R^{p}} k\left(\frac{\boldsymbol{x}-\boldsymbol{u}}{a_{j}}\right) h(\boldsymbol{u}) d \boldsymbol{u}\right]^{2} \\
=\int_{R^{p}} k^{2}(\boldsymbol{v}) g\left(\boldsymbol{x}-a_{j} \boldsymbol{v}\right) d \boldsymbol{v}-a_{j}^{p}\left[\int_{R^{p}} k(\boldsymbol{v}) h\left(\boldsymbol{x}-a_{j} \boldsymbol{v}\right) d \boldsymbol{v}\right]^{2} .
\end{gathered}
$$

Therefore,

$$
\begin{aligned}
\mid \frac{a_{n}^{p} \sigma_{n}^{2}}{n} & -\alpha g(\boldsymbol{x}) \int_{R^{p}} k^{2}(\boldsymbol{u}) d \boldsymbol{u} \mid \\
= & \int_{R^{p}} k^{2}(\boldsymbol{v})\left|\frac{1}{n} \sum_{j=1}^{n}\left(\frac{a_{n}}{a_{j}}\right)^{p} g\left(\boldsymbol{x}-a_{j} \boldsymbol{v}\right)-\alpha g(\boldsymbol{x})\right| d \boldsymbol{v} \\
& +\frac{a_{n}^{p}}{n} \sum_{j=1}^{n}\left[\int_{R^{p}} k(\boldsymbol{v}) h\left(\boldsymbol{x}-a_{j} \boldsymbol{v}\right) d \boldsymbol{v}\right]^{2} \\
\leqq & \int_{R^{p}} k^{2}(\boldsymbol{v})\left\{\frac{1}{n} \sum_{j=1}^{n}\left(\frac{a_{n}}{a_{j}}\right)^{p}\left|g\left(\boldsymbol{x}-a_{j} \boldsymbol{v}\right)-g(\boldsymbol{x})\right|\right\} d \boldsymbol{v} \\
& +g(\boldsymbol{x})\left|\frac{1}{n} \sum_{j=1}^{n}\left(\frac{a_{n}}{a_{j}}\right)^{p}-\alpha\right| \int_{R^{p}} k^{2}(\boldsymbol{v}) d \boldsymbol{v}+O\left(a_{n}^{p}\right) .
\end{aligned}
$$

The second and third terms of the last expression converge to 0 by (1.5) and by the fact that $a_{n} \rightarrow 0$ as $n \rightarrow \infty$. The first term is bounded above by (recall that $a_{1}>a_{2}>\cdots$ $\left.>a_{n}>\cdots>0\right)$

$$
\frac{1}{n} \sum_{j=1}^{n} \int_{R^{p}}\left|g\left(\boldsymbol{x}-a_{j} \boldsymbol{v}\right)-g(\boldsymbol{x})\right| k^{2}(\boldsymbol{v}) d \boldsymbol{v} .
$$

Since $g(\boldsymbol{x})=E\left(Y^{2} \mid \boldsymbol{X}=\boldsymbol{x}\right) f(\boldsymbol{x})$ is bounded and continuous at $\boldsymbol{x}$ and since $\int_{\boldsymbol{R}^{p}} k^{2}(\boldsymbol{v}) d \boldsymbol{v}$ $\leqq \sup _{\boldsymbol{y} \in R^{p}} k(\boldsymbol{y})<\infty$, it follows from the Lebesque Dominated Convergence Theorem that 


$$
\int_{R^{p}}\left|g\left(\boldsymbol{x}-a_{n} \boldsymbol{v}\right)-g(\boldsymbol{x})\right| k^{2}(\boldsymbol{v}) d \boldsymbol{v} \rightarrow 0
$$

as $n \rightarrow \infty$. With an application of Lemma A, this implies that (2.8) converges to 0 as $n \rightarrow \infty$, establishing (2.6), and hence (2.3). Assertion (2.4) can be proved similarly.

Our next task is to establish the (weak and strong) uniform consistency for $\hat{m}(\boldsymbol{x})$.

LEMMA 3. Assume that conditions of Lemma 1 hold. If $h$ is a bounded function and $Y$ is a bounded random variable, then

$$
\sup _{\boldsymbol{x} \in R p}|E \hat{h}(\boldsymbol{x})-h(\boldsymbol{x})| \rightarrow 0 \quad \text { as } n \rightarrow \infty .
$$

PROOF. Note that $a_{j}^{-p} E Y_{j} k\left[\left(\boldsymbol{x}-\boldsymbol{X}_{j}\right) / a_{j}\right]=\int_{R^{p}} h\left(\boldsymbol{x}-a_{j} \boldsymbol{v}\right) k(\boldsymbol{v}) d \boldsymbol{v}$ which is uniformly bounded (since $h$ is). In view of Lemma A, the lemma will be established if we can show that

$$
\sup _{\boldsymbol{x} \in R^{p}}\left|\frac{1}{a_{n}} E Y_{n} k\left(\frac{\boldsymbol{x}-\boldsymbol{X}_{n}}{a_{n}}\right)-h(\boldsymbol{x})\right| \rightarrow 0 \quad \text { as } n \rightarrow \infty .
$$

Now, for a given $\delta>0$, partition $R^{p}=A \cup A^{c}$, where

$$
A=\{\boldsymbol{u}:\|\boldsymbol{u}\| \leqq \delta\}=\left\{\boldsymbol{v}:\|\boldsymbol{v}\| \leqq \delta / a_{n}^{p}\right\} .
$$

Then the left hand side of (2.9) is equal to

$$
\begin{aligned}
\sup _{\boldsymbol{x} \in R^{p}} \int_{R^{p}} \mid & h\left(\boldsymbol{x}-a_{n} \boldsymbol{v}\right)-h(\boldsymbol{x}) \mid k(\boldsymbol{v}) d \boldsymbol{v} \leqq \sup _{\boldsymbol{x} \in R^{p}}\left(\int_{A}+\int_{A^{c}}\right) \\
\leqq & \sup _{\boldsymbol{x} \in R^{p}} \sup _{\boldsymbol{u} \in A}|h(\boldsymbol{x}-\boldsymbol{u})-h(\boldsymbol{x})| \\
& +\frac{a_{n}^{p}}{\delta} \sup _{\boldsymbol{v} \in A}[\|\boldsymbol{v}\| k(\boldsymbol{v})] \sup _{\boldsymbol{x} \in R^{p}}\left\{\int_{A^{c}}\left|h\left(\boldsymbol{x}-a_{n} \boldsymbol{v}\right)\right| d \boldsymbol{v}+|h(\boldsymbol{x})| \int_{A^{c}} d \boldsymbol{v}\right\} .
\end{aligned}
$$

Since $a_{n} \rightarrow 0, \delta / a_{n}^{p} \rightarrow \infty$ (i. e., $A^{c} \rightarrow \phi$ ) as $n \rightarrow \infty$, the fact that $h$ is uniformly bounded together with Condition (1.2) implies that both terms in the braces of the last expression converge to 0 as $n$ tends to $\infty$. Now, (2.9) is established by letting $\delta \rightarrow 0$.

LEMma 4. Assume that conditions of Lemma 2 hold. If $f$ and $h$ are bounded, if

$$
n a_{n}^{2 p} \rightarrow \infty,
$$

and if the characteristic function of $k, \phi(\boldsymbol{t})=\int_{R^{p}} e^{i t^{\prime} \boldsymbol{x}} k(\boldsymbol{x}) d \boldsymbol{x}$, is absolutely integrable and nondecreasing in the negative part and nonincreasing in the positive part for each argument, then

in probability, as $n \rightarrow \infty$.

$$
\sup _{\boldsymbol{x} \in R}|\hat{h}(\boldsymbol{x})-h(\boldsymbol{x})| \rightarrow 0,
$$

Proof. The proof is similar to that of Theorem 4 of Yamato (1971). From Lemma 3 it suffices to show that

$$
\sup _{\boldsymbol{x} \in R p}|\hat{h}(\boldsymbol{x})-E \hat{h}(\boldsymbol{x})| \rightarrow 0,
$$

in probability, as $n \rightarrow \infty$. Since $\phi(t)$ and $k(\boldsymbol{u})$ are absolutely integrable, we have 


$$
\begin{aligned}
& k(\boldsymbol{x})=\frac{1}{(2 \pi)^{p}} \int_{R^{p}} e^{-i t^{\prime} \boldsymbol{x}} \phi(\boldsymbol{t}) d \boldsymbol{t} \\
& \hat{h}(\boldsymbol{x})=\frac{1}{(2 \pi)^{p}} \int_{R^{p}}\left[\frac{1}{n} \sum_{j=1}^{n} Y_{j} e^{i t^{\prime} \boldsymbol{x}_{j}} \phi\left(a_{j} \boldsymbol{t}\right)\right] e^{-i t^{\prime} \boldsymbol{x}} d \boldsymbol{t}
\end{aligned}
$$

and

$$
E \hat{h}(\boldsymbol{x})=\frac{1}{(2 \pi)^{j}} \int_{R^{p}}\left[\frac{1}{n} \sum_{j=1}^{n} \phi\left(a_{j} \boldsymbol{t}\right)\right] \psi(\boldsymbol{t}) e^{-i \boldsymbol{t}^{\prime} \boldsymbol{x}} d \boldsymbol{t}
$$

where $\phi(\boldsymbol{t})$ is the Fourier transform of $h(\boldsymbol{x})$. Hence

$$
\begin{aligned}
& E\left[\sup _{\boldsymbol{x} \boxminus R^{p}}|\hat{h}(\boldsymbol{x})-E \hat{h}(\boldsymbol{x})|\right] \\
& \quad \leqq \frac{1}{(2 \pi)^{p}} E \int_{R^{p}}\left|\frac{1}{n} \sum_{j=1}^{n}\left\{Y_{j} e^{i \boldsymbol{t}^{\prime} \boldsymbol{x}_{j}}-\phi(\boldsymbol{t})\right\} \phi\left(a_{j} \boldsymbol{t}\right)\right| d \boldsymbol{t} \\
& \quad \leqq \frac{1}{(2 \pi)^{p}} \int_{R^{p}}\left\{\frac{1}{n^{2}} \sum_{j=1}^{n} E\left|Y_{j} e^{i^{\prime \prime} \boldsymbol{x}_{j}}-\phi(\boldsymbol{t})\right|^{2}\left|\phi\left(a_{j} \boldsymbol{t}\right)\right|^{2}\right\}^{1 / 2} d \boldsymbol{t} .
\end{aligned}
$$

The last expressioh in (2.12) follows by an application of Schwarz's inequality. Note that, for all $j=1, \cdots, n, E\left|Y_{j} e^{i t^{\prime} x_{j}}-\psi(\boldsymbol{t})\right|^{2} \leqq C^{2}<\infty$, uniformly in $\boldsymbol{t}$. This fact together with the assumptions on $\phi(t)$ and (2.10) implies that (2.12) is further bounded above by

$$
\frac{C}{(2 \pi)^{p} n} \int_{R^{p}}\left\{n\left|\phi\left(a_{n} \boldsymbol{t}\right)\right|^{2}\right\}^{1 / 2} d \boldsymbol{t}=\frac{C}{(2 \pi)^{p}\left(n a_{n}^{2 p}\right)^{1 / 2}} \int_{R^{p}}|\phi(\boldsymbol{u})| d \boldsymbol{u},
$$

which converges to 0 as $n$ tends to $\infty$. The Assertion (2.11) follows by an application of Markov's inequality.

The weak uniform consistency of $\hat{m}(\boldsymbol{x})$ is established in the following:

THEOREM 3. Assume that conditions of Lemma 4 hold. Let $B$ be a proper closed subset of $R^{p}$ such that $\inf _{\boldsymbol{x} \in B} f(\boldsymbol{x})=\mu>0$ and $\sup _{\boldsymbol{x} \in B}|m(\boldsymbol{x})|=\nu<\infty$. Then

$$
\sup _{\boldsymbol{x} \in B}|\hat{m}(\boldsymbol{x})-m(\boldsymbol{x})| \rightarrow 0 \text {. }
$$

in probability, as $n \rightarrow \infty$.

PROOF. Note that, for all $n$ sufficiently large, we have

$$
\begin{array}{r}
\sup _{\boldsymbol{x} \in B}|\hat{m}(\boldsymbol{x})-m(\boldsymbol{x})| \leqq \sup _{\boldsymbol{x} \in B}\left|\frac{\hat{h}(\boldsymbol{x})}{\hat{f}(\boldsymbol{x})}-\frac{h(\boldsymbol{x})}{\hat{f}(\boldsymbol{x})}\right|+\sup _{\boldsymbol{x} \in B}\left|\frac{h(\boldsymbol{x})}{\hat{f}(\boldsymbol{x})}-\frac{h(\boldsymbol{x})}{f(\boldsymbol{x})}\right| \\
\leqq \frac{1}{\inf _{\boldsymbol{x} \in B} \hat{f}(\boldsymbol{x})}\left\{\sup _{\boldsymbol{x} \in B}|\hat{h}(\boldsymbol{x})-h(\boldsymbol{x})|+\sup _{\boldsymbol{x} \in B}|m(\boldsymbol{x})| \sup _{\boldsymbol{x} \in B}|\hat{f}(\boldsymbol{x})-f(\boldsymbol{x})|\right\} .
\end{array}
$$

It is known that, (see Yamato (1971), Theorem 4) under the assumptions of the theorem,

$$
\sup _{\boldsymbol{x} \in R^{p}}|\hat{f}(\boldsymbol{x})-f(\boldsymbol{x})| \rightarrow 0,
$$

in probability, as $n \rightarrow \infty$. This implies that

$$
\inf _{\boldsymbol{x} \in B} \hat{f}(\boldsymbol{x}) \geqq \mu-\varepsilon>0 .
$$

Thus, for all $n$ sufficiently large, we have 


$$
\sup _{\boldsymbol{x} \in B}|\hat{m}(\boldsymbol{x})-m(\boldsymbol{x})| \leqq \frac{1}{\mu-\varepsilon}\left\{\sup _{\boldsymbol{x} \in B}|\hat{h}(\boldsymbol{x})-h(\boldsymbol{x})|+\nu \sup _{\boldsymbol{x} \in B}|\hat{f}(\boldsymbol{x})-f(\boldsymbol{x})|\right\},
$$

which converges to 0 , in probability, as $n \rightarrow \infty$, by Lemma 4 and (2.13).

To prove the strong uniform consistency of $\hat{m}(\boldsymbol{x})$ over the subset $B$ as defined in Theorem 3, in view of Inequality (2.14), it is sufficient to establish the strong uniform consistency for $\hat{f}(\boldsymbol{x})$ and $\hat{h}(\boldsymbol{x})$. The result is stated here without proof.

THEOREM 4. Let $B$ be a proper closed subset of $R^{p}$ such that $\inf _{\boldsymbol{x} \in B} f(\boldsymbol{x})=\mu>0$ and $\sup _{\boldsymbol{x} \in B}|m(\boldsymbol{x})|=\nu<\infty$. If , under some regularity conditions,

$$
\sup _{\boldsymbol{x} \in B}|\hat{f}(\boldsymbol{x})-f(\boldsymbol{x})| \rightarrow 0, \quad \text { w.p. } 1,
$$

and

$$
\sup _{\boldsymbol{x} \in B}|\hat{h}(\boldsymbol{x})-h(\boldsymbol{x})| \rightarrow 0, \quad w . p .1,
$$

as $n \rightarrow \infty$, then

$$
\sup _{\boldsymbol{x} \in B}|\hat{m}(\boldsymbol{x})-m(\boldsymbol{x})| \rightarrow 0, \quad w . p .1,
$$

as $n \rightarrow \infty$.

REMARK. Davies (1973) showed under the assumptions that $k(\boldsymbol{u})$ satisfies Conditions (1.1) and (1.2), that $\phi(\boldsymbol{t})=\int_{R^{p}} e^{i t^{\prime} \boldsymbol{x}} k(\boldsymbol{x}) d \boldsymbol{x}$ is absolutely integrable, and that $\left\{a_{n}\right\}$ satisfies (1.3) and the following conditions,

$$
\begin{aligned}
a_{n} a_{n+1}^{-1} \rightarrow 1 & \text { and } n a_{n}^{-2 p} \rightarrow \infty, \quad \text { as } n \rightarrow \infty, \\
& \sum_{n=1}^{\infty}\left(n a_{n}^{p}\right)^{-2}<\infty, \quad \text { and } \\
& \sum_{n=1}^{\infty}\left(n a_{n}^{2 p-1}\right)^{-1}\left(a_{n+1}^{-1}-a_{n}^{-1}\right)<\infty .
\end{aligned}
$$

Then, if $f$ is uniformly continuous on $B$, (2.15) holds. It is observed that if, in addition to the above assumptions, $Y$ is bounded and $h$ is uniformly continuous on $B$ with absolutely integrable Fourier transform, then (2.16) also holds. This can be shown using Davies' arguments.

\section{Joint asymptotic distribution of the regression estimate at a finite number of distinct points.}

Let $\boldsymbol{x}_{1}, \cdots, \boldsymbol{x}_{q}$ be $q$ distinct points of $f(\boldsymbol{x})$ in $R^{p}$. Under some regularity conditions the joint asymptotic distribution of the random vector $\left[\hat{m}\left(\boldsymbol{x}_{1}\right), \cdots, \hat{m}\left(\boldsymbol{x}_{q}\right)\right]$ will be derived. Though the theorem is proved only for the special case when $q=2$, it is clear that the method of proof remains valid in the more general case. The results presented in this section are generalizations over those of Schuster (1972) since (i) $m(\boldsymbol{x})$ is now a multiple regression and (ii) $\hat{m}(\boldsymbol{x})$ is a sequential estimate. Though the proofs bear some resemblance to those of Schuster (1972), there are major difference which need to be clarified. To simplify the presentation we will follow the same procedures and define similar notation as those in Schuster. For $j=1, \cdots, n, s=1,2$, 
let

$$
\begin{array}{ll}
U_{j}^{*}\left(\boldsymbol{x}_{s}\right)=a_{j}^{-p} k\left[\left(\boldsymbol{x}_{s}-\boldsymbol{X}_{j}\right) / a_{j}\right], & U_{j}\left(\boldsymbol{x}_{s}\right)=a_{i}^{p / 2}\left[U_{j}^{*}\left(\boldsymbol{x}_{s}\right)-E U_{j}^{*}\left(\boldsymbol{x}_{s}\right)\right] \\
V_{j}^{*}\left(\boldsymbol{x}_{s}\right)=Y_{j} U_{i}^{*}\left(\boldsymbol{x}_{s}\right) & V_{j}\left(\boldsymbol{x}_{s}\right)=a_{j}^{p / 2}\left[V_{j}^{*}\left(\boldsymbol{x}_{s}\right)-E V_{j}^{*}\left(\boldsymbol{x}_{s}\right)\right] \\
\bar{U}_{n}\left(\boldsymbol{x}_{s}\right)=\sum_{j=1}^{n} U_{j}\left(\boldsymbol{x}_{s}\right), & \bar{V}_{n}\left(\boldsymbol{x}_{s}\right)=\sum_{j=1}^{n} V_{j}\left(\boldsymbol{x}_{s}\right) \\
W_{j}=\left[U_{j}\left(\boldsymbol{x}_{1}\right), U_{j}\left(\boldsymbol{x}_{2}\right), V_{j}\left(\boldsymbol{x}_{1}\right),\right. & \left.V_{j}\left(\boldsymbol{x}_{2}\right)\right]^{\prime},
\end{array}
$$

and

$$
n^{1 / 2} Z_{n}=\left[\bar{U}_{n}\left(\boldsymbol{x}_{1}\right), \bar{U}_{n}\left(\boldsymbol{x}_{2}\right), \bar{V}_{n}\left(\boldsymbol{x}_{1}\right), \bar{V}_{n}\left(\boldsymbol{x}_{2}\right)\right]^{\prime} .
$$

We will establish the asymptotic normality of $\boldsymbol{Z}_{n}$ by showing that

$$
\frac{\sum_{j=1}^{n} E\left|\boldsymbol{C}^{\prime} \boldsymbol{W}_{j}\right|^{3}}{n^{3 / 2}\left(\operatorname{Var} \boldsymbol{C}^{\prime} \boldsymbol{Z}_{n}\right)^{3 / 2}}=o(1),
$$

for any real vector $C=\left(c_{1}, c_{2}, d_{1}, d_{2}\right)^{\prime}$ in $R^{4}$. (See, Loève (1963), page 275). To evaluate the denominator of (3.1), write

$$
\begin{aligned}
\sigma_{n}^{2}= & \operatorname{Var}\left(\boldsymbol{C}^{\prime} \boldsymbol{Z}_{n}\right) \\
= & \frac{1}{n}\left\{c_{1}^{2} \operatorname{Var} \bar{U}_{n}\left(\boldsymbol{x}_{1}\right)+c_{2}^{2} \operatorname{Var} \bar{U}_{n}\left(\boldsymbol{x}_{2}\right)+d_{1}^{2} \operatorname{Var} \bar{V}_{n}\left(\boldsymbol{x}_{1}\right)+d_{2}^{2} \operatorname{Var} \bar{V}_{n}\left(\boldsymbol{x}_{2}\right)\right. \\
& +2 c_{1} c_{2} \operatorname{cov}\left[\bar{U}_{n}\left(\boldsymbol{x}_{1}\right), \bar{U}_{n}\left(\boldsymbol{x}_{2}\right)\right]+2 c_{1} d_{1} \operatorname{cov}\left[\bar{U}_{n}\left(\boldsymbol{x}_{1}\right), \bar{V}_{n}\left(\boldsymbol{x}_{1}\right)\right] \\
& +2 c_{1} d_{2} \operatorname{cov}\left[\bar{U}_{n}\left(\boldsymbol{x}_{1}\right), \bar{V}_{n}\left(\boldsymbol{x}_{2}\right)\right]+2 c_{2} d_{1} \operatorname{cov}\left[\bar{U}_{n}\left(\boldsymbol{x}_{2}\right), \bar{V}_{n}\left(\boldsymbol{x}_{1}\right)\right] \\
& \left.+2 c_{2} d_{2} \operatorname{cov}\left[\bar{U}_{n}\left(\boldsymbol{x}_{2}\right), \bar{V}_{n}\left(\boldsymbol{x}_{2}\right)\right]+2 d_{1} d_{2} \operatorname{cov}\left[\bar{V}_{n}\left(\boldsymbol{x}_{1}\right), \bar{V}_{n}\left(\boldsymbol{x}_{2}\right)\right]\right\} .
\end{aligned}
$$

The above variances and covariances can be evaluated under the assumptions that

$$
\begin{gathered}
\partial f / \partial x_{i}, \partial h / \partial x_{i}, \partial g / \partial x_{i} \quad(i=1, \cdots, p) \quad \text { exist and bounded, and } \\
\int_{R^{p}} \boldsymbol{u} k(\boldsymbol{u}) d \boldsymbol{u}=0, \quad \int_{R^{p}}\|\boldsymbol{u}\|^{2} k(\boldsymbol{u}) d \boldsymbol{u}<\infty .
\end{gathered}
$$

They turn out to be as follows: For $s=1,2$,

$$
\begin{gathered}
\frac{1}{n} \operatorname{Var} \bar{U}_{n}\left(\boldsymbol{x}_{s}\right)=f\left(\boldsymbol{x}_{s}\right) \int_{R^{p}} k^{2}(\boldsymbol{u}) d \boldsymbol{u}+O\left(a_{n}\right), \\
\frac{1}{n} \operatorname{Var} \bar{V}_{n}\left(\boldsymbol{x}_{s}\right)=g\left(\boldsymbol{x}_{s}\right) \int_{R^{p}} k^{2}(\boldsymbol{u}) d \boldsymbol{u}+O\left(a_{n}\right), \\
\frac{1}{n} \operatorname{cov}\left[\bar{U}_{n}\left(\boldsymbol{x}_{s}\right), \bar{V}_{n}\left(\boldsymbol{x}_{s}\right)\right]=h\left(\boldsymbol{x}_{s}\right) \int k^{2}(\boldsymbol{u}) d \boldsymbol{u}+O\left(a_{n}\right), \\
\frac{1}{n} \operatorname{cov}\left[\bar{U}_{n}\left(\boldsymbol{x}_{1}\right), \bar{V}_{n}\left(\boldsymbol{x}_{2}\right)\right]=O\left(a_{n}\right), \\
\frac{1}{n} \operatorname{cov}\left[\bar{U}_{n}\left(\boldsymbol{x}_{1}\right), \bar{U}_{n}\left(\boldsymbol{x}_{2}\right)\right]=O\left(a_{n}\right), \\
\frac{1}{n} \operatorname{cov}\left[\bar{U}_{n}\left(\boldsymbol{x}_{s}\right), \bar{V}_{n}\left(\boldsymbol{x}_{\boldsymbol{s}}\right)\right]=O\left(a_{n}\right) .
\end{gathered}
$$


We will sketch the proofs for (3.4) and (3.5). For (3.4) note that

Then

$$
\begin{aligned}
& \left|\operatorname{Var} U_{j}(\boldsymbol{x})-f(\boldsymbol{x}) \int k^{2}(\boldsymbol{u}) d \boldsymbol{u}\right| \\
& \quad \leqq \int\left|f\left(\boldsymbol{x}-a_{j} \boldsymbol{u}\right)-f(\boldsymbol{x})\right| k^{2}(\boldsymbol{u}) d \boldsymbol{u}+a_{j}^{p}\left[\int f\left(\boldsymbol{x}-a_{j} \boldsymbol{u}\right) k(\boldsymbol{u}) d \boldsymbol{u}\right]^{2} \\
& \quad=O\left(a_{j}\right)+O\left(a_{j}^{\boldsymbol{p}}\right)=O\left(a_{j}\right) .
\end{aligned}
$$

$$
\begin{aligned}
& \left|\frac{1}{n} \operatorname{Var} \bar{U}_{n}(\boldsymbol{x})-f(\boldsymbol{x}) \int k^{2}(\boldsymbol{u}) d \boldsymbol{u}\right| \\
& \quad \leqq \frac{1}{n} \sum_{j=1}^{n}\left|\operatorname{Var} U_{j}(\boldsymbol{x})-f(\boldsymbol{x}) \int k^{2}(\boldsymbol{u}) d \boldsymbol{u}\right|=O\left(\eta_{n}\right),
\end{aligned}
$$

where

$$
\eta_{n}=\frac{1}{n} \sum_{j=1}^{n} a_{j}
$$

It follows from Lemma A that $a_{n} \rightarrow 0$ implies $\eta_{n} \rightarrow 0$ but not conversely. Therefore $O\left(\eta_{n}\right) \leqq O\left(a_{n}\right)$, verifying (3.4). As for (3.5), note that, for $\boldsymbol{x}_{1} \neq \boldsymbol{x}_{2}$,

$$
\begin{aligned}
\mid a_{j}^{p} \operatorname{cov}[ & \left.U_{j}^{*}\left(\boldsymbol{x}_{1}\right), V_{j}^{*}\left(\boldsymbol{x}_{2}\right)\right] \mid \\
\leqq & a_{j}^{p} \mid \int a_{j}^{-2 n} Y_{j} k\left(\frac{\boldsymbol{x}_{1}-\boldsymbol{u}}{a_{j}}\right) k\left(\frac{\boldsymbol{x}_{2}-\boldsymbol{u}}{a_{j}}\right) f(\boldsymbol{u}) d \boldsymbol{u} \\
& \quad-\int a_{j}^{-p} k\left(\frac{\boldsymbol{x}_{1}-\boldsymbol{u}}{a_{j}}\right) f(\boldsymbol{u}) d \boldsymbol{u} \int a_{j}^{-p} Y_{j} k\left(\frac{\boldsymbol{x}_{2}-\boldsymbol{u}}{a_{j}}\right) f(\boldsymbol{u}) d \boldsymbol{u} \mid \\
\leqq & \left|\int k(\boldsymbol{v}) k\left(\frac{\boldsymbol{x}_{2}-\boldsymbol{x}_{1}}{a_{j}}+\boldsymbol{v}\right) h\left(\boldsymbol{x}_{1}-a_{j} \boldsymbol{v}\right) d \boldsymbol{v}\right| \\
& +a_{j}^{p} \int k(\boldsymbol{v}) f\left(\boldsymbol{x}_{1}-a_{j} \boldsymbol{v}\right) d \boldsymbol{v}\left|\int k(\boldsymbol{v}) h\left(\boldsymbol{x}_{2}-a_{j} \boldsymbol{v}\right) d \boldsymbol{v}\right| \\
= & \left|\int k(\boldsymbol{v}) k\left(\frac{\boldsymbol{x}_{2}-\boldsymbol{x}_{1}}{a_{j}}+\boldsymbol{v}\right) h\left(\boldsymbol{x}_{1}-a_{j} \boldsymbol{v}\right) d \boldsymbol{v}\right|+O\left(a_{j}^{p}\right) .
\end{aligned}
$$

It will be shown that the first term of the last expression converges to 0 as $a_{j} \rightarrow 0$, i. e.,

$$
a_{j}^{-1}\left|\int_{R^{p}} k(\boldsymbol{v}) k\left(\frac{\boldsymbol{x}_{2}-\boldsymbol{x}_{1}}{a_{j}}+\boldsymbol{v}\right) h\left(\boldsymbol{x}_{1}-a_{j} \boldsymbol{v}\right) d \boldsymbol{v}\right|=o(1) .
$$

Assume that

$$
\sup _{\boldsymbol{x} \in R^{p}}|h(\boldsymbol{x})| \leqq \sup _{\boldsymbol{x} \in R^{p}} E(|Y| \mid \boldsymbol{x})<\infty,
$$

(which is implied by the assumption that $E\left(|Y|^{3} \mid \boldsymbol{x}\right)<\infty$ for all $\boldsymbol{x} \in R^{p}$ ). Then the integrand in (3.7) is bounded above by $k(\boldsymbol{v}) \cdot \sup _{\boldsymbol{y} \in R^{p}} k(\boldsymbol{y}) \cdot \sup _{\boldsymbol{x} \in R}|h(\boldsymbol{x})|$ which, of course, is integrable. Thus, by the Lebesque Dominated Convergence Theorem, the quantity in (3.7) converges to

$$
\left|h\left(\boldsymbol{x}_{1}\right)\right| \int_{R^{p}} k(\boldsymbol{v}) \lim _{a_{j} \rightarrow 0}\left[a_{j}^{-1} k\left(\frac{\boldsymbol{x}_{2}-\boldsymbol{x}_{1}}{a_{j}}+\boldsymbol{v}\right)\right] d \boldsymbol{v}=0
$$

due to Condition (1.2). This establishes (3.7). Now 


$$
\begin{aligned}
\frac{1}{n}\left|\operatorname{cov}\left[\bar{U}_{n}\left(\boldsymbol{x}_{1}\right), \bar{V}_{n}\left(\boldsymbol{x}_{2}\right)\right]\right| & \leqq \frac{1}{n} \sum_{j=1}^{n} a_{j}^{p}\left|\operatorname{cov}\left[U_{j}^{*}\left(\boldsymbol{x}_{1}\right), V_{j}^{*}\left(\boldsymbol{x}_{2}\right)\right]\right| \\
& =\frac{1}{n} \sum_{j=1}^{n} O\left(a_{j}\right)=O\left(\eta_{n}\right),
\end{aligned}
$$

where $\eta_{n}$ is given by (3.6). It is clear $O\left(\eta_{n}\right) \leqq O\left(a_{n}\right)$, establishing (3.5). Hence,

$$
\sigma_{n}^{2}=\int_{R^{p}} k^{2}(\boldsymbol{u}) d \boldsymbol{u} \sum_{s=1}^{2}\left[c_{s}^{2} f\left(\boldsymbol{x}_{s}\right)+d_{s}^{2} g\left(\boldsymbol{x}_{s}\right)+2 c_{s} d_{s} h\left(\boldsymbol{x}_{s}\right)\right]+O\left(a_{n}\right) .
$$

To evaluate the numerator on the left hand side of (3.1), put $\rho_{n j}^{3}=n^{-3 / 2} E\left|\boldsymbol{C}^{\prime} \boldsymbol{W}_{j}\right|^{3}$ and $\rho_{n}^{3}=\sum_{j=1}^{n} \rho_{n j}^{3}$ so that

$$
\begin{aligned}
\rho_{n}^{3} \leqq & 16 n^{-3 / 2} \sum_{j=1}^{n}\left(\left|c_{1}\right|{ }^{3} E\left|U_{j}\left(\boldsymbol{x}_{1}\right)\right|^{3}+\left|c_{2}\right|^{3} E\left|U_{j}\left(\boldsymbol{x}_{2}\right)\right|^{3}\right. \\
& \left.+\left|d_{1}\right|^{3} E\left|V_{j}\left(\boldsymbol{x}_{1}\right)\right|^{3}+\left|d_{2}\right|^{3} E\left|V_{j}\left(\boldsymbol{x}_{2}\right)\right|^{3}\right) \\
\leqq & 16 n^{-3 / 2}\left(\left|c_{1}\right|^{3}+\left|c_{2}\right|^{3}+\left|d_{1}\right|^{3}+\left|d_{2}\right|^{3}\right) \max _{s=1,2}\left[E\left|U_{j}\left(\boldsymbol{x}_{s}\right)\right|^{3}, E\left|V_{j}\left(\boldsymbol{x}_{s}\right)\right|^{3}\right] .
\end{aligned}
$$

However, for $s=1,2$, and all $j$ sufficiently large, we have

and

$$
\begin{aligned}
E\left|U_{j}\left(\boldsymbol{x}_{s}\right)\right|^{3} & \cong a_{j}^{-3 p / 2} E\left|k\left(\frac{\boldsymbol{x}_{s}-\boldsymbol{X}_{j}}{a_{j}}\right)\right|^{3} \\
& =a_{j}^{-p / 2}\left|\int_{R^{p}} k^{3}(\boldsymbol{u}) f\left(\boldsymbol{x}_{s}-a_{j} \boldsymbol{u}\right) d \boldsymbol{u}\right| \\
& =O\left(a_{j}^{-p / 2}\right),
\end{aligned}
$$

$$
E\left|V_{j}\left(\boldsymbol{x}_{s}\right)\right|^{3} \cong a_{j}^{-p / 2}\left|\int k^{3}(\boldsymbol{u}) h^{*}\left(\boldsymbol{x}_{s}-a_{j} \boldsymbol{u}\right) d \boldsymbol{u}\right|=O\left(a_{j}^{-p / 2}\right),
$$

provided that

$$
h^{*}(\boldsymbol{x})=E\left(Y^{3} \mid \boldsymbol{X}=\boldsymbol{x}\right) f(\boldsymbol{x})<\infty
$$

for all $\boldsymbol{x} \in R^{p}$. Therefore,

$$
\begin{aligned}
\rho_{n}^{3} & \leqq 16 n^{-3 / 2} \sum_{j=1}^{n} O\left(a_{j}^{-p / 2}\right) \\
& =O\left(\frac{1}{n} \sum_{j=1}^{n}\left(n a_{j}^{p}\right)^{-1 / 2}\right) \leqq O\left(\left(n a_{n}^{p}\right)^{-1}\right),
\end{aligned}
$$

by an application of Lemma A. Thus $\rho_{n} \rightarrow 0$ as $n \rightarrow \infty$ provided that Condition (1.3) holds. Therefore $\rho_{n} / \sigma_{n} \rightarrow 0$ as $n \rightarrow \infty$ for any $\boldsymbol{C} \neq \mathbf{0}$, establishing (3.1). We have proved the following result:

LemMA 5. Suppose that $k$ satisfies Conditions (1.1), (1.2), and (3.3). Assume that $E\left(|Y|^{3} \mid \boldsymbol{X}=\boldsymbol{x}\right)<\infty$ for all $\boldsymbol{x} \in R^{p}$ and that (3.2) holds. If $\boldsymbol{x}_{1} \neq \boldsymbol{x}_{2}, f\left(\boldsymbol{x}_{s}\right)>0, s=1,2$, then $\boldsymbol{Z}_{n}$ is asymptotically normally distributed with mean vector $\boldsymbol{0} \in R^{4}$ and covariance matrix

$$
\Gamma=\int_{R^{p}} k^{2}(\boldsymbol{u}) d \boldsymbol{u}\left[\begin{array}{cccc}
f\left(\boldsymbol{x}_{1}\right) & h\left(\boldsymbol{x}_{1}\right) & 0 & 0 \\
h\left(\boldsymbol{x}_{1}\right) & g\left(\boldsymbol{x}_{1}\right) & 0 & 0 \\
0 & 0 & f\left(\boldsymbol{x}_{2}\right) & h\left(\boldsymbol{x}_{2}\right) \\
0 & 0 & h\left(\boldsymbol{x}_{2}\right) & g\left(\boldsymbol{x}_{2}\right)
\end{array}\right] .
$$


The next lemma gives a set of sufficient conditions on $f, h$, and $a_{n}$ such that $E \hat{f}(\boldsymbol{x})$ and $E \hat{h}(\boldsymbol{x})$ in $\boldsymbol{Z}_{n}$ may be replaced by $f(\boldsymbol{x})$ and $h(\boldsymbol{x})$, respectively.

LemMa 6. Suppose that assumptions of Lemma 5 hold. If Condition (1.4) holds and if $\partial^{2} f / \partial x_{i} \partial x_{j}, \partial^{2} h / \partial x_{i} \partial x_{j}(i, j=1, \cdots, p)$ exist and are bounded, then

$$
\begin{gathered}
\boldsymbol{Z}_{n}^{*}=n^{-1 / 2} \sum_{j=1}^{n} a_{j}^{p / 2}\left[U_{j}^{*}\left(\boldsymbol{x}_{1}\right)-f\left(\boldsymbol{x}_{1}\right), V_{j}^{*}\left(\boldsymbol{x}_{1}\right)-h\left(\boldsymbol{x}_{1}\right),\right. \\
\left.U_{j}^{*}\left(\boldsymbol{x}_{2}\right)-f\left(\boldsymbol{x}_{2}\right), V_{j}^{*}\left(\boldsymbol{x}_{2}\right)-h\left(\boldsymbol{x}_{2}\right)\right]^{\prime}
\end{gathered}
$$

has the same joint asymptotic normal distribution as that of $\boldsymbol{Z}_{n}$.

PROOF. It suffices to show that the $i$-th component of $Z_{n}^{*}$ has the same limiting marginal distribution as that of the $i$-th component of $\boldsymbol{Z}_{n}$ for $i=1,2,3,4$. Since

$$
\boldsymbol{Z}_{n}-\boldsymbol{Z}_{n}^{*}=n^{-1 / 2} \sum_{j=1}^{n} a_{j}^{p / 2} \boldsymbol{B}_{j}
$$

where $\boldsymbol{B}_{j}=\left(b_{1 j}, b_{2 j}, b_{3 j}, b_{4 j}\right)^{\prime}$ with

$$
\begin{array}{ll}
b_{1 j}=f\left(\boldsymbol{x}_{1}\right)-E U_{j}^{*}\left(\boldsymbol{x}_{1}\right), & b_{2 j}=h\left(\boldsymbol{x}_{1}\right)-E V_{j}^{*}\left(\boldsymbol{x}_{1}\right), \\
b_{3 j}=f\left(\boldsymbol{x}_{2}\right)-E U_{j}^{*}\left(\boldsymbol{x}_{2}\right), & b_{4 j}=h\left(\boldsymbol{x}_{2}\right)-E V_{j}^{*}\left(\boldsymbol{x}_{2}\right),
\end{array}
$$

the lemma will be proved if we can show that

$$
n^{-1 / 2} \sum_{j=1}^{n} a_{j}^{p / 2}\left|b_{i j}\right|=\frac{1}{n} \sum_{j=1}^{n}\left(n a_{j}\right)^{p / 2}\left|b_{i j}\right| \rightarrow 0 \quad \text { as } n \rightarrow \infty
$$

for $i=1, \cdots, 4$. But, by the Taylor expansion of $f\left(\boldsymbol{x}_{1}-a_{j} \boldsymbol{u}\right)$ about $\boldsymbol{x}_{1}$ and the asymption (3.3), we have

$$
\left.\left|b_{1 j}\right|=\mid \int_{R^{p}}\left[f\left(\boldsymbol{x}_{1}\right)-f\left(\boldsymbol{x}_{1}-a_{j} \boldsymbol{u}\right)\right] k(\boldsymbol{u}) d \boldsymbol{u}\right] \mid \leqq O\left(a_{j}^{2}\right) .
$$

Similarly, $\left|b_{i j}\right|=O\left(a_{j}^{2}\right)$, for $i=2,3,4$. Now, applying Lemma A, we have

$$
\frac{1}{n} \sum_{j=1}^{n}\left(n a_{j}^{p}\right)^{1 / 2}\left|b_{i j}\right| \leqq \frac{1}{n} \sum_{j=1}^{n} O\left(\left(n a_{j}^{p+4}\right)^{1 / 2}\right),
$$

which converges to 0 by (1.4) and Lemma A.

Now we are in a position to state the main theorem of this section, whose proof follows directly from Lemmas 5 and 6 along with Theorem (ii) of Rao (1965, page 321).

THEOREM 5. Suppose that $k$ satisfies Conditions (1.1), (1.2) and (3.3). Let $\partial f / \partial x_{i}$, $\partial h / \partial x_{i}, \partial g / \partial x_{i}, \partial^{2} f / \partial x_{i} \partial x_{j}, \partial^{2} h / \partial x_{i} \partial x_{j}(i, j=1, \cdots, p)$ all exist and be bounded. Let $\left\{a_{n}\right\}$ satisfy (1.3) and (1.4). If $\boldsymbol{x}_{i} \neq \boldsymbol{x}_{j}, f\left(\boldsymbol{x}_{i}\right)>0(i \neq j=1, \cdots, q)$, then

$$
\left(n a_{n}^{p}\right)^{1 / 2}\left[\hat{m}\left(\boldsymbol{x}_{1}\right)-m\left(\boldsymbol{x}_{1}\right), \cdots, \hat{m}\left(\boldsymbol{x}_{q}\right)-m\left(\boldsymbol{x}_{q}\right)\right]^{\prime}
$$

is asymptotically normally distributed with mean vector $0 \in R^{q}$ and diagonal covariance matrix $\Sigma=\left(\sigma_{i j}\right)$ where $\sigma_{i i}=\operatorname{Var}\left(Y \mid \boldsymbol{X}=\boldsymbol{x}_{i}\right) \int_{R^{p}} k^{2}(\boldsymbol{u}) d \boldsymbol{u} / f\left(\boldsymbol{x}_{i}\right), i=1, \cdots, q$.

REMARKS. 1. To the best of our knowledge the joint asymptotic distribution of $\left[m_{n}\left(\boldsymbol{x}_{1}\right), \cdots, m_{n}\left(\boldsymbol{x}_{q}\right)\right]$ is not documented in the literature, where $m_{n}(\boldsymbol{x})$ is the nonsequential estimate given by (1.8). However, it can be shown that, under the assumptions of Theorem $1, m_{n}(\boldsymbol{x}) \rightarrow m(\boldsymbol{x})$, in probability as $n \rightarrow \infty$. This implies that $\hat{m}(\boldsymbol{x})$ and 
$m_{n}(\boldsymbol{x})$ have the same limiting distribution. Therefore, the random vector $\left[m_{n}\left(\boldsymbol{x}_{1}\right), \cdots\right.$, $\left.m_{n}\left(\boldsymbol{x}_{q}\right)\right]$ has the same limiting distribution as that of $\left[\hat{m}\left(\boldsymbol{x}_{1}\right), \cdots, \hat{m}\left(\boldsymbol{x}_{q}\right)\right]$.

2. Conditions on the sequence $\left\{a_{n}\right\}$ imposed in Theorem 5 when $p=1$ agree with those of Schuster (1972) except Condition (1.3). For $p=1$, Condition (1.3) reduces to $n a_{n} \rightarrow \infty$, as $n \rightarrow \infty$. Instead of this, Schuster has $n a_{n}^{3} \rightarrow \infty$, as $n \rightarrow \infty$. This is mainly due to different assumptions on the moment of $|Y|^{3}$. Schuster assumes that $E|Y|^{3}<\infty$, while we assume $E\left(|Y|^{3} \mid \boldsymbol{X}=\boldsymbol{x}\right)<\infty$ for all $\boldsymbol{x} \in R^{p}$. Had we assumed $E|Y|^{3}<\infty$, Condition (1.3) would have been

$$
n a_{n}^{3 p} \rightarrow \infty, \quad \text { as } n \rightarrow \infty .
$$

However, there is no such $\left\{a_{n}\right\}$ that satisfies both Conditions (3.10) and (1.4) except for $p=1$.

\section{References}

[1] G. BenNett (1962), Probability Inequalities for the Sum of Independent Random Vari. ables, J. Amer. Statist. Assoc., Vol. 57, pp. 33-45.

[2] T. CACoullos (1966), Estimation of a Multivariate Density, Ann. Inst. Statist. Math., Vol. 18, pp. 179-189.

[3] H.I. Davies (1973), Strong Consistency of a Sequential Estimator of a Probability Density Function, Bull. Math. Statist., Vol. 16, pp. 49-54.

[4] Nadaraya (1964), On Estimating Regression, Theory Prob. Appl., Vol. 9, pp. 141-142.

[5] C.R. RAO (1965), Linear Statistical Inference and Its Applications, John Wiley and Sons, N.Y.

[6] E.F. Schuster (1972), Joint Asymptotic Distribution of the Estimated Regression at a Finite Number of Distinct Points, Ann. Math. Statist., Vol. 43, pp. 84-88.

[7] J. VanRyzin (1969), On Strong Consistency of Density Estimates, Ann. Math. Statist., Vol. 40, pp. 1765-1772.

[8] G.S. Watsok (1964), Smooth Regression Analysis, Sankhya Ser. A, Vol. 26, pp. 359-372.

[9] H. Yamato (1971), Sequential Estimation of a Continuous Probability Density Function and Mode, Bull. Math. Statist., Vol. 14, pp. 1-12. 